This item was submitted to Loughborough's Research Repository by the author.

Items in Figshare are protected by copyright, with all rights reserved, unless otherwise indicated.

\title{
Sensor selection in neuro-fuzzy modelling for fault diagnosis
}

PLEASE CITE THE PUBLISHED VERSION

http://dx.doi.org/10.1109/ISIE.2010.5637885

PUBLISHER

(c) IEEE

VERSION

VoR (Version of Record)

LICENCE

CC BY-NC-ND 4.0

REPOSITORY RECORD

Zhou, Yimin, and Argyrios C. Zolotas. 2019. "Sensor Selection in Neuro-fuzzy Modelling for Fault Diagnosis". figshare. https://hdl.handle.net/2134/7737. 
This item was submitted to Loughborough's Institutional Repository (https://dspace.lboro.ac.uk/) by the author and is made available under the following Creative Commons Licence conditions.

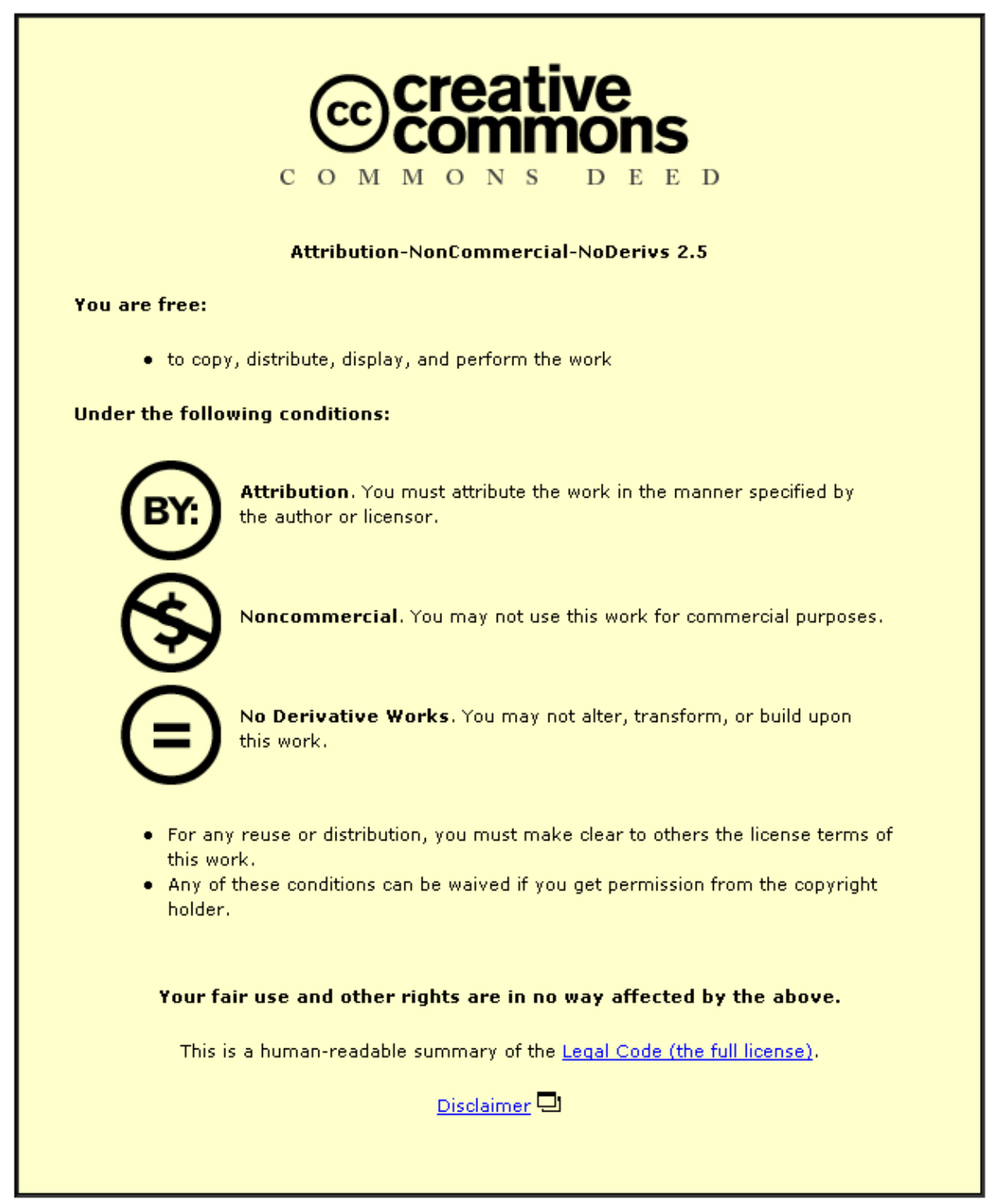

For the full text of this licence, please go to: http://creativecommons.org/licenses/by-nc-nd/2.5/ 


\title{
Sensor Selection in Neuro-fuzzy Modelling for Fault Diagnosis
}

\author{
Yimin Zhou, Argyrios Zolotas \\ Control Group, Department of Electronic \& Electrical Engineering, \\ Loughborough University, Loughborough, LE11 3TU \\ E-mail:y.zhou2@lboro.ac.uk,a.c.zolotas@lboro.ac.uk
}

\begin{abstract}
In this paper, sensor selection relating to neurofuzzy modeling for the purpose of fault diagnosis is discussed. The input/output selection in fuzzy modelling plays an important role in the performance of the derived model. In addition, with respect to fault tolerant issues, the impact of the faults on the system, i.e. possible incipient and abrupt faults, should be detected in the earliest possible instance. The paper first presents a brief introduction to neuro-fuzzy modelling, and proceeds to sensor selection with the aim of considerably improving the quality and reliability of the system. We study faults, both of abrupt and incipient nature, that can be diagnosed in an immediate sense. A two-tank system is used as an example to illustrate the studied concepts.
\end{abstract}

\section{INTRODUCTION}

With the development of high-technique and precision equipment, industrial systems are becoming more complicated and consist of nonlinear models in the design process. Undoubtedly, sensor measurements play a significant role in fault diagnosis and fault tolerant control since they carry useful information for the measured (or monitored) signals both for control and fault tolerance purposes (subject to requirements).

Interestingly, the issue of sensor selection for control and fault tolerance is still an open research area with challenging issues to study and methods to develop. In this context there is no generic sensor selection framework for the case of selecting sensors for control and fault tolerance (something that has been pointed out recently in 2003 by the IFAC TC on SafeProcess regarding design of systems with given level of reliability).

Albeit, there are methodologies for selecting sensor/actuator pairs for improving controllability/ observability indexes mainly in large flexible structures [1], as well as sensor selection in sensor networks (but in this case the problem is with regards to fusion of information). However, the aforementioned methods are different to the ones considered in the research related to this paper as the current one looks at selection of sensors in the context of closed loop control with fault tolerance capability (or equivalently design of closed loop control with given degree of reliability). In particular, the paper presents some preliminary results in the area of fusing sensor selection to fuzzy modeling for nonlinear systems.

Traditional physical or mathematical modeling techniques are sometimes not able to describe the nonlinear operational behaviour properly in large-scale systems. Fuzzy modeling or neuro-fuzzy modeling is therefore adopted for nonlinear model identification as a black-box or grey-box alternative. Neuro-fuzzy modeling includes the selection of input, output and identification algorithm to determine the relationship between input and output of the system. The model performance of describing the system behaviour depends largely on the quality of the training data. Muller and his cowork discusses criteria for controllability and observability in [8]. The characteristics of the derived system, such as observability, controllability, degree of stability could be used as criteria to evaluate the possible sensor sets for fault detection issues and further decision making expositions. Sensor selection has been discussed in linear systems with optimal control [1], and LMI formulations [6]. With the aid of $\mathrm{H}_{2} / \mathrm{H}_{\infty}$ controller design metrics, different sensor sets were tested to obtain the optimal performance/minimum cost of output estimation (OE) in the total cost (TC). Note that these (and related) methods are application (and controller) dependent.

Fuzzy logic with some issues of sensor selection is discussed in [5]. Redundant sensor information is normally used in the system with high precision, reliability and security requirements. Hence, output measurements are assessed before entering the fuzzy modelling procedure. Four methods in two architectures for sensor validation and fusion are illustrated. Crisp determination and fuzzy fitness with a weight on sensor validation are also discussed. Also, sensor selection in process monitoring is discussed in [12], With fuzzy set theory used for the decision of sensor suitability due to specific requirements. The weight of each sensor is calculated by the membership function under certain requirements and the weight of the requirements is also evaluated.

This paper, studies an approach of evaluating sensor sets for detecting faults in the system in the context of nonlinear systems with black-box neuro-fuzzy modelling. A nonlinear two-tank system is used for illustrating elements of the proposed approach and a next step in the classification of sensor sets for control. 
The remainder of the paper is as follows. Section II gives the introduction of fuzzy modelling technique and the identification procedure. Methods of sensor selection in fuzzy modelling for fault diagnosis are discussed in section III. Simulation experiments and results with a two-tank water level system are illustrated in section IV. Finally, conclusions and future work are given in section $\mathrm{V}$.

\section{NEURO-FUZZY MODELLING}

\section{A. Introduction of Neuro-fuzzy Mmodelling}

The identification of a neuro-fuzzy model mainly consists of three phases: Structure Identification, Parameter Identification and Model Evaluation [13]. Identifying the correct structure of the model is the first step. Diverse types of parameters are demanded for the structure selection: shapes of membership functions, $A N D$ and $O R$ operations, implications, defuzzification methods, consequence types, etc. The other important factor during this stage is the determination of the model order, which also involves the construction of the rule base. The performance of the model is largely dependent on the training data. In order to obtain the training data set, experiments should be designed so that the input signals are maximally excited at the normal operational conditions of the actual plant [2].

Increasing the number of free parameters in a model structure, improves the fitting of the training data. A neural network model should be flexible enough to approximate any nonlinear function to a given (required) accuracy. At the same time, it should also try to avoid over-parameterisation due to the inherent noise in most practical training data sets. In addition, any data which produces abnormal results should be eliminated from the training data set. The number and distribution of the fuzzy sets of the input/output variables also have an effect on the accuracy of model and should be selected carefully.

Note that in the case of unstable systems these can be first stabilized with some appropriate (simple if possible) controller and tests performed on the stabilized system.

\section{B. Fuzzy Relational Model Identification}

Fuzzy relational model (FRM) is used as an example to illustrate the modelling process, whose diagram is shown in Fig 1. Consider a multi-input and multi-output (MIMO) system consisting of $n$ inputs $\left(x_{1}, x_{2}, \cdots, x_{n}\right)$ and $m$ output $\left(y_{1}, y_{2}, \cdots, y_{m}\right)$, where the input and output spaces are characterized by $r_{1}, r_{2}, \cdots, r_{n}$ and $j_{1}, j_{2}, \cdots, j_{m}$ fuzzy reference sets, respectively. The FRM is a predefined set of linguistic rules, each of which has an associated rule confidence and it can be described by the following fuzzy relational equation:

$$
Y_{j}(k)=X_{1}(k) \bullet X_{2}(k) \bullet \cdots \bullet X_{n}(k) \bullet R
$$

where $Y_{j}(k)(j=1, \cdots, m)$ and $X_{i}(k)(i=1, \cdots, n)$ are fuzzy representations of the elements of output $y_{j}(k)$ and inputs $x_{i}(k)$ at the sampling instant $k$; precisely, they delegate the firing degree of the output $y_{j}$ input $x_{i}$ in the selected membership function. $R$ is the fuzzy relational matrix which stores the values of the rule confidences representing the relationship between the inputs and outputs. "•" denotes fuzzy compositional inference. The rule confidence, $R_{r_{1} r_{2} \cdots r_{n}, j}$ indicates the amount of confidence of the output $y_{j}(k)$ when inputs are $x_{1, s_{1}}, x_{2, s_{2}}, \cdots, x_{n, s_{n}},\left(1 \leq s_{1} \leq r_{1}\right.$, $1 \leq s_{2} \leq r_{2}, \cdots, 1 \leq s_{n} \leq r_{n}$ ) . Normally, fuzzy sets are partitioned by triangle or trapezoidal membership function. Global Recursive Least Squares (GRLS) algorithm as one of the identification algorithms is employed to estimate the rule confidences $R$ [7].

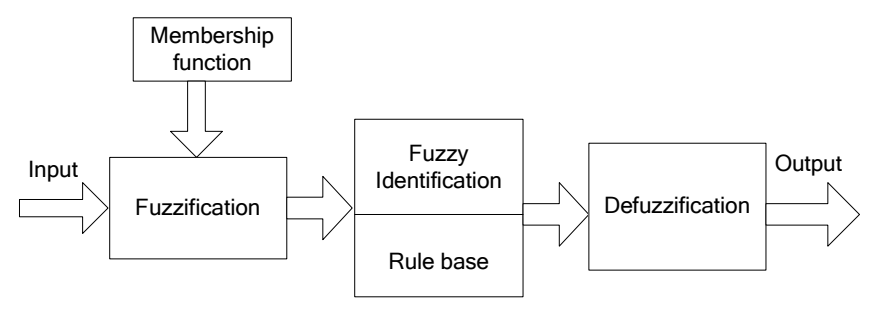

Fig 1 Diagram of fuzzy modelling

A satisfactory fuzzy relational model can only be obtained if the training data are complete, accurate and noise-free. Simulation results have shown that different sets of training data can result in various prediction quality of fuzzy models [11]. Therefore, acquiring high quality of the training data is a key issue in generating an accurate fuzzy relational model[14].

\section{METHOD OF SENSOR SELECTION IN NEURO-FUZZY MODELING}

As mentioned previously in the paper, the area of sensor selection is interesting and challenging especially targeting towards practical engineering applications. Interesting work has been presented by Sowers et al in their paper [10] but from the point of view of selecting sensors for diagnostic purposes on health monitoring of an aerospace system. Their study concentrates on the application of model-based technique on statistical evaluation techniques some being application dependent and some non-application dependent. Their choice of sensors depends on the results of fault detectability. Note that there is no specific concern of closed loop control as such.

Moreover, there is a number of issues to consider in sensor selection either for control, monitoring or fault tolerant capability. Increasing complexity of system structures brings in larger number of sensor elements with a heavier burden on financial issues in the system as well as reliability issues. For example, simply looking at the number of sensors to consider in designing a system with some questions in the following: (i) how many sensors to use for control (if not only monitoring), which relates to what is required to control in the system, (ii) the controller structure to implement, (iii) only few sensors provide simplicity in the design process but 
is it enough from the performance point of view? (iv) incorporating more information by using extra sensors introduces more possibilities of sensor faults, thus what is the relationship to implementation and maintainability. Some of these have been considered in a specific application of magnetic levitation by Michail et al in their works, i.e. refer to [9].

In the context of this paper, we are investigating the sensor selection with neuro-fuzzy modelling issues of nonlinear systems. The assumption is that an excitation signal is provided to the process (this can be either a reference, feedback or controlled variable) with possible sensor sets evaluated in terms of the level of performance in detecting faults in the system. A statistical approach is followed, due to assuming a random test signal exciting the process, i.e. metrics of Root Mean Square of the error between actual and estimated output information as well as the use of Integral Time Absolute Error which is more appropriate for cases of incipient fault scenario.

\section{A. Fault Detection with Neuro-Fuzzy Model}

Consider a system with three inputs $\left(x_{1}, x_{2}, x_{3}\right)$ and four outputs $\left(y_{1}, y_{2}, y_{3}, y_{4}\right)$, in fact even if the model is MIMO it can be decomposed into a number of MISO equivalents. For example, a MISO first-order model is used for model identification i.e., consider three input and one output variables in the model, as shown in Fig 2. The selection of the input variables can be based on monitoring or control purposes (i.e. can be states or measured outputs in the system). In this case, we illustrate the process with $x_{1}(k-1), x_{2}(k-1)$ and $y_{1}(k-1)$ (feedback of output 1 used for estimation purposes). The output is $y_{1}(k)$.

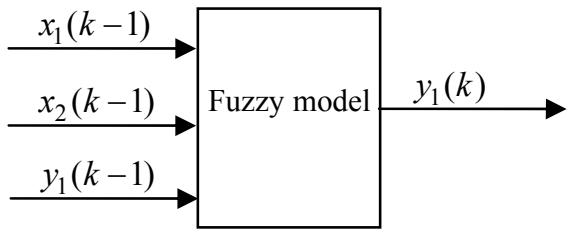

Fig 2 The structure of the fuzzy model

The structure of the evaluation for fault diagnosis includeing elements of dedicated observer related schemes [4] is shown in Fig 3, with the example referring to sensor $y_{1}$.

In Fig 3, $f_{s}$ is the possible sensor fault; $y_{1}$ is one of the system outputs; $\hat{y}_{1(M 1)}$ and $\hat{y}_{1(M 2)}$ are output estimates of the aforementioned sensors from two derived models M1 and M2. In fact, M1 and M2 are considered same models trained off-line with no modelling errors. The previous system output $y_{1}(k-1)$ is regarded as one of the inputs to predict the current output $\hat{y}_{1}(k)$ in $\mathbf{M} 1$ and $\hat{y}_{1(M 2)}(k-1)$ is used in M2 instead (note the feedback of this estimate which may be used for online updating of the models if necessary).

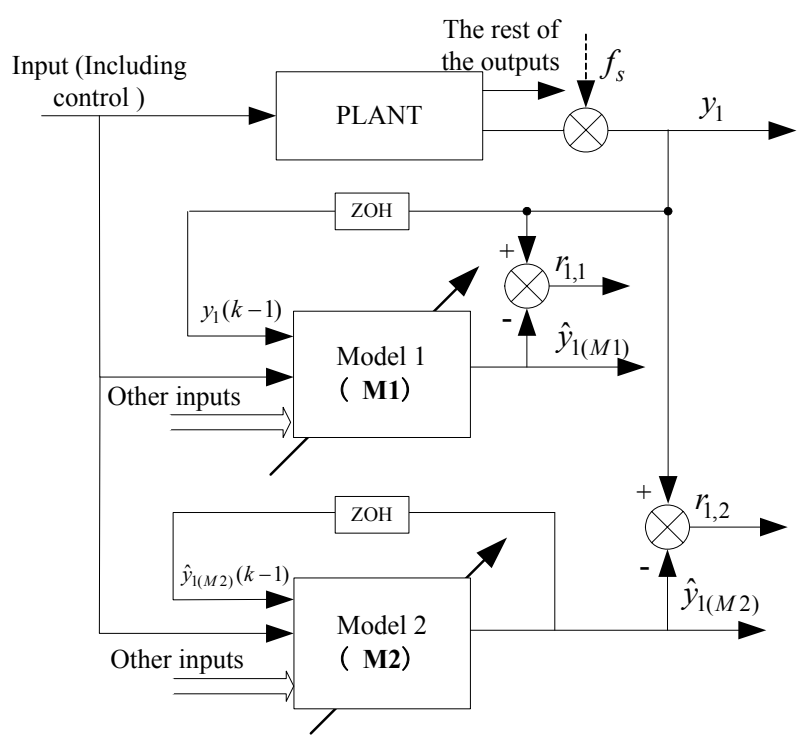

Fig 3 The structure of the fault diagnosis system

The sampling time can be decided based on the application characteristics. If there is no sensor fault in the system, the model would produce the predicted output in the normal range of the value. The error $e_{y_{1}}$ between the outputs $\hat{y}_{1}(k-1)$ and $\hat{y}_{1(M 1)}(k)$ in two continuous sampling moments is given by,

$$
e_{y_{1(M 1)}}=\left|y_{1}(k-1)-\hat{y}_{1(M 1)}(k)\right| \leq \delta
$$

where $\delta$ is the pre-defined threshold at the sampling moments. At the same time, the previous output $\hat{y}_{1(M 2)}(k-1)$ in $\mathbf{M} 2$ is treated as one of the inputs to predict the current output $\hat{y}_{2(M 2)}(k)$. The error between the previous and current outputs from M2 is,

$$
e_{y_{1(M 2)}}=\left|\hat{y}_{1(M 2)}(k-1)-\hat{y}_{1(M 2)}(k)\right|
$$

which can be used for fault detection. Under normal operation all errors should be small, however, if there is a sensor fault in $y_{1}$ then (assuming no model update) M1 will mistakenly provide a small residual while $\mathbf{M} 2$ should be able to identify the fault and raise a flag for that purpose, i.e.

$$
\left\|e_{y_{1(M 1)}}\right\| \gg\left\|e_{y_{1(M 2)}}\right\| \text {. }
$$

Once a fault is detected in the system, measures will be taken to accommodate this situation. One solution is to switch to different controllers to reduce or remove the effect of the fault in the system, or guarantee level of robustness due to setpoint changes (i.e. model mismatch) in case of no model updates (this is more critical in processes with fast dynamics).

This fault diagnosis scheme is similar to the dedicated observer scheme for fault detection. It can be extended to incorporate further dedicated models (observers) relating to more outputs (i.e. introduce coping with extra sensor faults) of interest and if necessary introduce a data fusion 
mechanism in later steps. Therefore, the detection and isolation of faults can be achieved simultaneously. Once a sensor measurement is identified as faulty, it will be detected and replaced by an alternative set.

It is worth noting that in the case of subsystem fault, the design of controller is essential to guarantee robustness and in the first instance avoid (usually computationally intensive) model updates (as mentioned though this is most critical in fast changing dynamics applications).

\section{B. Sensor Selection in Neuro-fuzzy Modelling of Fault Diagnosis}

Consider $n$ sensor outputs in the system, if we consider the selection of each sensor from a binary point of view as 0 : not selected, 1: selected, a total of $\left(2^{n}-1\right)$ possible sensor sets arise. For example, with 4 possible sensors one has 15 possible combinations (note that at least one sensor should be selected). Besides the control input signals, all of the measurements could be included in the input to the neurofuzzy model.

However, one important issue with the utilisation of neuro-fuzzy modelling is the number of inputs to the model. As discussed in [15] and references within, increasing number of rules in the model could result in increasing the number of rules in the rule base. Albeit, the model performance could be improved, with more information available in the model. Since adding one more input, the rule number of the model will be increased to $n_{j}$ times of the original rule number, where $n_{j}$ is the fuzzy set number of the added input one. The input set should be selected carefully.

All of the combinations of the sensor measurements combined with the controlled inputs are used as input set in the developed models. The models are evaluated to identify the most appropriate input (to the model) combinations. These possible sets can be evaluated through the performance of the developed model. Root mean squared error (RMSE) is used here as a simple first criterion to evaluate the resulting performance, i.e.

$$
R M S E=\sqrt{\frac{1}{N} \sum_{i=1}^{N}\left(y_{i}-\hat{y}_{i}\right)^{2}}
$$

where, $N$ is the data number; $y_{i}$ and $\hat{y}_{i}$ are the system output and model output respectively. An additional metric is used, that of the Integral-(of)-Time-(multiplied)-Absolute-Error (ITAE), which introduces the weighting of time on the error

$$
\text { ITAE }=\int_{0}^{\infty} t|e(t)| d t
$$

where $e$ is the error between the system output and model output (targeting possible incipient fault cases, with details to be presented in the final version of the paper). The selection procedure can de summarized as follows:

(1) Define the sensor sets;

(2) Train the model with the above potential input sets;

(3) Validate the developed model performances with RMSE and ITAE;

(4) Evaluate the sensor sets in the input sets of the models;
(5) Choose the sets with best performance.

\section{CASE STUDY - TWO WATER-TANK SYSTEM}

An example of a water level control system, seen in Fig 5, is used to describe the procedure of fuzzy relational modeling and sensor selection for fault diagnosis. Although the twotank system is rather simple, it is helpful to illustrate the procedure.

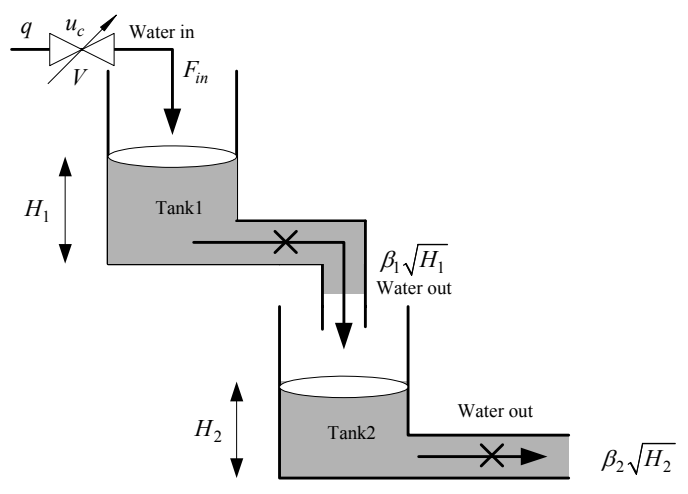

Fig 5 Schematic diagram for the water-tank process system

With the assumption that the liquid density remains constant, the nonlinear equations for the height of water in the tank, $H_{1}, H_{2}$, are given by

$$
\begin{aligned}
& A \frac{d H_{1}}{d t}+\beta_{1} \sqrt{H_{1}}=F_{i n} \\
& A \frac{d H_{2}}{d t}+\beta_{2} \sqrt{H_{2}}=\beta_{1} \sqrt{H_{1}} \\
& F_{\text {in }}=q \cdot u_{c}
\end{aligned}
$$

where $F_{\text {in }}$ is the volumetric flow rate into Tank1, which is controlled by the valve $V$; The influx of water depends linearly on the input variables $u_{c} \in[0,1]$, and $q$ is the inlet flow rate. $A$ is the cross-sectional area of the tank; $\beta_{1}$ and $\beta_{2}$ are the valve coefficients. The height of $H_{2}$ is a secondorder nonlinear differential equation and the model for this water tank system is shown below:

$$
H_{2}(n)=f\left(u(n-1), \beta_{1}(n-1), H_{1}(n-1), H_{2}(n-1)\right)
$$

where $f(\bullet)$ is a nonlinear function for the relationship between input and output; $u(n-1), \beta_{1}(n-1), H_{1}(n-1)$ and $H_{2}(n-1)$ are the input and $H_{2}(n)$ is the output of the model. System parameter settings can be referred in [3]. The training data set is generated with the method described in [14].

\section{A. Fault-free case}

As it is known, there are $\left(2^{n}-1\right)$ possible sensor sets with $n$ sensors in the system. Here, we assume three possible sensors, i.e. measuring heights in Tank 1 and Tank 2 ([s1],[s2] respectively) and measuring valve position between Tank 1 and Tank 2 ([s3]). One has $\left(2^{3}-1\right)=7$ possible 
sensor sets for the design process and these are, $\{[s 1],[s 2],[s 3],[s 1, s 2],[s 2, s 3],[s 1, s 3],[s 1, s 2, s 3]\}$. It is assumed that the measurement of height Tank 2 is the system requirement, and at this point sets including that above sensor are emphasised $\{\mathrm{A} 1, \mathrm{~A} 2, \mathrm{~A} 3, \mathrm{~A} 4\}$ with, $\{A 1:[s 2], A 2:[s 1, s 2]$, $A 3:[s 2, s 3], A 4:[s 1, s 2, s 3]\}$. Therefore, there are four possible input sets to be selected for the neuro-fuzzy model. All four models are described as:

$$
\begin{aligned}
& \hat{H}_{2}(n)=f_{1}\left(u(n-1), H_{2}(n-1)\right) \\
& \hat{H}_{2}(n)=f_{2}\left(u(n-1), H_{1}(n-1), H_{2}(n-1)\right) \\
& \hat{H}_{2}(n)=f_{3}\left(u(n-1), H_{2}(n-1), \beta_{1}(n-1)\right) \\
& \hat{H}_{2}(n)=f_{4}\left(u(n-1), H_{1}(n-1), H_{2}(n-1), \beta_{1}(n-1)\right)
\end{aligned}
$$

There are 5 fuzzy unevenly division for the input signal $u(n-1)$ in the fuzzy reference space considering the response relationship between the control $u$ and the height $H_{2}$. As for the inputs $H_{1}(n-1)$ and $H_{2}(n-1)$, there are 5 evenly fuzzy sets assigned in the fuzzy reference spaces. Three fuzzy sets are assigned for the input signal $\beta_{1}(n-1)$. Therefore, the rule numbers of the four models are: $25,125,75$, and 375 respectively. The model performance is tested by a random excitation test signal. This random test signal will be used for all following tests. One of the test results with which model developed with sensor set A3 is shown in Fig 6. Note that currently fault-free case is considered.

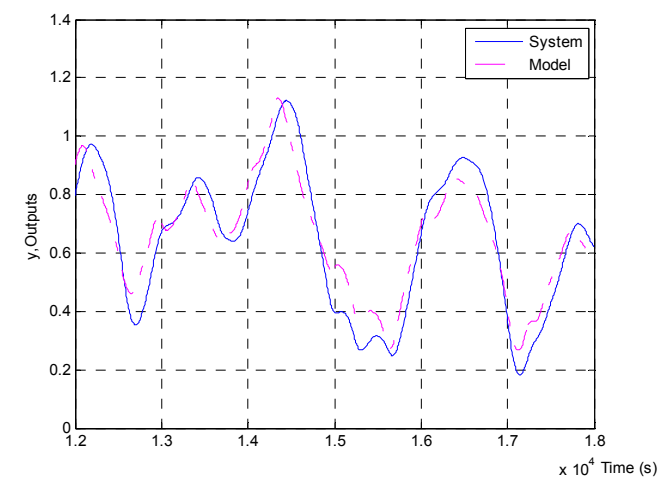

Fig 6 The performance of the developed model

The root mean squared error (RMSE) between the output from system and models could be used as a criterion to evaluate the set performance. It has to be mentioned that the estimated output of the model will be used as the input for the model test, which has the same structure as described in Fig 3 of the input for model M2. The RMSE of the developed models is shown in Table 1.

The difference between Test 1 and Test 2 is the use of estimated $\hat{H}_{1}(n-1)$ as one of the inputs to the model (when the relative sensor exists to compare) in the second test, while the actual measurement $H_{1}(n-1)$ is used as one of the inputs to the model in the first test. Referring to Test 1 , it can be seen that the RMSE is decreasing with more inputs added to the model. However, the RMSE is fluctuating with more inputs in Test 2 (issue with sensor [s1], illustrating also the interest in selecting the type of sensor, in such case there is deficiency in the estimate of $\left.\hat{H}_{1}(n-1)\right)$. In this fault-free case, one might decide not to include the sensor for the measurement of $H_{1}(n-1)$.

TABLE I

THE RMSE OF THE POTENTIAL SENSOR SETS

\begin{tabular}{|c|c|c|c|c|c|}
\hline \multirow{2}{*}{ Test } & Model & A1 & A2 & A3 & A4 \\
\cline { 2 - 2 } & Fuzzy set & $(5 \times 5)$ & $(5 \times 5 \times 5)$ & $(5 \times 5 \times 3)$ & $(5 \times 5 \times 5 \times 3)$ \\
\hline \multirow{2}{*}{1} & $\begin{array}{c}\text { M1, } \\
H_{1}(n-1), \hat{H}_{2}(n-1)\end{array}$ & 0.1683 & $\mathbf{0 . 1 2 2 6}$ & 0.0723 & $\mathbf{0 . 0 1 4 7}$ \\
\hline 2 & $\hat{H}_{1}(n-1), \hat{H}_{2}(n-1)$ & 0.1683 & 0.4156 & 0.0723 & 0.2581 \\
\hline
\end{tabular}

Another group of models is developed in Test 3, still faultfree case, with reduced fuzzy sets on one of the inputs to the model, i.e., $H_{2}(n-1)$, from 5 down to 3 (i.e. reducing rule base complexity). The RMSE of the developed models is shown in Table 2, with the estimated value $\hat{H}_{1}(n-1)$ regarded as one of the inputs to the model. The Model trained with the set A4 which includes all sensors has the best performance, followed by the one in A3, while A2 has the worst. However, here there is some improvement to the RMSE compared to Test 2 due to the relaxation of the rule base. Even in this case one still could decide on not utilising $H_{1}(n-1)$, also sensor set A3 still provides enough information for a small RMSE thus one could use this set to avoid an extra sensor and thus reduce both the possible extra sensor fault instance and cost in the implementation. (as well as reducing the complexity and computational burden).

TABLE 2

THE RMSE OF THE POTENTIAL SENSOR SETS

\begin{tabular}{|c|c|c|c|}
\hline Test & $\begin{array}{c}\mathrm{A} 2 \\
(5 \times 3 \times 3)\end{array}$ & $\begin{array}{c}\mathrm{A} 3 \\
(5 \times 3 \times 3)\end{array}$ & $\begin{array}{c}\mathrm{A} 4 \\
(5 \times 3 \times 3 \times 3)\end{array}$ \\
\hline 3 & 0.1515 & 0.0645 & 0.024 \\
\hline
\end{tabular}

\section{B. Faulty cases}

After the sensor set in the input of neurofuzzy model has been decided, fault diagnosis is tested with the developed model in this case with sensor set A3. Two types of sensor faults for sensor [s2] are tested in the fault diagnosis scheme. One is incipient fault (slow change fault development in the measurement of the height) and another is abrupt fault (a pulse-type fluctuation). The incipient sensor fault occurs after running for 5000s. As it is shown in Fig 7, the output from model M2 has a largely noticeable difference compared to the output from the system and the model M1. The fault can be detected shortly after its occurrence. As for the abrupt fault, it can be detected immediately when it occurs. The simulation result is shown in Fig 8. 

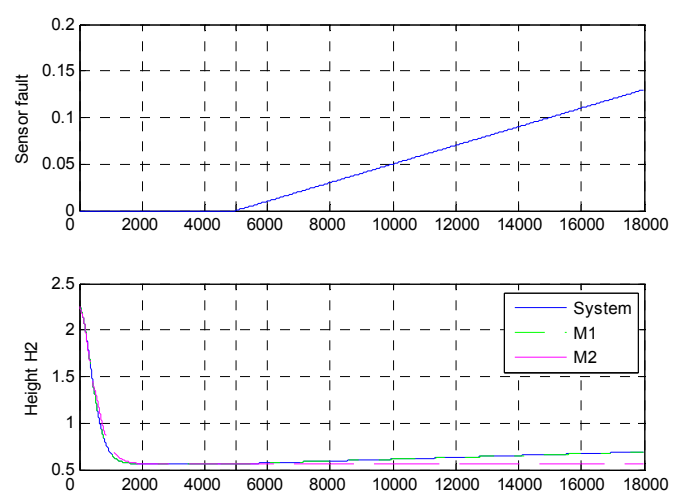

Fig 7 Fault diagnosis with incipient sensor fault
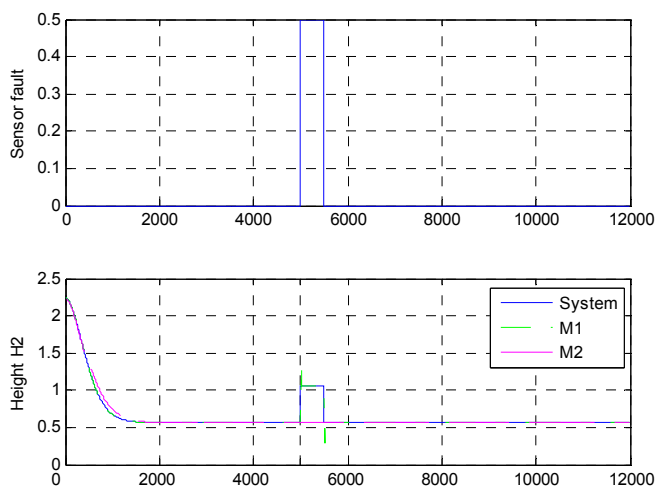

Fig 8 Fault diagnosis with abrupt sensor fault

When a component (and it happens to refer to the sensor [s3] due to the inclusion of this measurement) fault occurs in the system, i.e., the valve between the Tank1 and Tank 2 is accidentally at fully open position from its normal position. In simulation, the coefficient $\beta_{1}$ will be set with pulse-type fluctuation, i.e. the fault occurs at $10000 \mathrm{~s}$ and lasts $5000 \mathrm{~s}$. The responses from the system and two models with abrupt fault are shown in Fig 9.

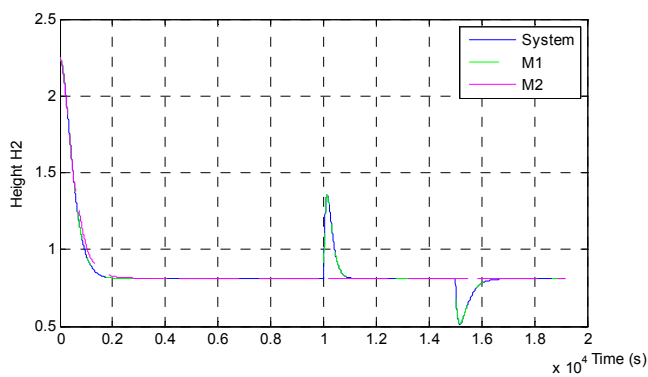

Fig 9 Height $\mathrm{H} 2$ response with component fault

In particular, when more sensor sets are involved and in the case of control design in the context of closed-loop system an iterative scheme should be followed in terms of selecting, tuning (using GA technique) and evaluating, suggestions appear in [9].

\section{CONCLUSION AND FUTURE WORK}

In this paper, sensor selection with neurofuzzy model is discussed. Based on given requirements in the system, sets of sensors are selected in the context of neuro-fuzzy modelling with issues of fault diagnosis. Cases of sensor faults have been studied. Results can be extended to actuator faults and component faults. Current research work investigates the fusion of sensor information in the proposed structures with closed-loop control design and reliability levels.

\section{ACKNOWLEDGMENT}

Part of this research is supported under the EPSRC PROJECT Grant Ref. EP/D063965/1 and BAE Systems. Y. Zhou would also like to thank Prof. Arthur Dexter of Oxford University for the useful discussions on issues of neuro-fuzzy modelling.

\section{REFERENCES}

[1] Balas, G. J. and P. M. Young (1999). "Sensor selection via closed-loop control objectives." IEEE Transactions on Control Systems Technology 7(6): 692-705.

[2] Costa, B. P. J. and J. A. Dente (2001). "Fuzzy systems modeling in practice." Fuzzy Sets and Systems 121(1): 73-93.

[3] Hussain, M. A. and P. Y. Ho (2004). "Adaptive sliding mode control with neural network based hybrid models." Journal of Process Control 14(2): 157-176.

[4] Isermann, R., (2005), Model-based fault-detection and diagnosis - status and applications, Annual Reviews in Control, Volume 29, Issue 1, 2005, Pages 71-85, ISSN 1367-5788.

[5] Lee, M. F. R., K. Stanley, et al. (2001). Implementation of sensor selection and fusion using fuzzy logic, Vancouver, BC, Canada, Institute of Electrical and Electronics Engineers Inc.

[6] Li, Z., A. C. Zolotas, et al. (2007). Output selection under control and fault detectability considerations. Control \& Automation, 2007. MED '07. Mediterranean Conference on.

[7] Ljung, L. (1983). Theory and practice of recursive identification, Cambridge, MassachusettsLondon, England : The MIT Press.

[8] Mueller, P. C. and H. I. Weber (1972). "Analysis And Optimization Of Certain Qualities Of Controllability And Observability For Linear Dynamical Systems." Automatica 8(3): 237-246.

[9] Michail, K., Zolotas, A.C. and Goodall, R.M., (2009). EMS systems: optimised sensor configurations for control and sensor fault tolerance. International Symposium on Speed-up, Safety and Service Technology for Railway and Maglev Systems (STECH'09), Niigata JAPAN, 6-19th June.

[10] Sowers, T. S., G. Kopasakis, et al. (2008). Application of the systematic sensor selection strategy for turbofan engine diagnostics, Berlin, Germany, American Society of Mechanical Engineers.

[11] Tan, W. W., Dexter, L. A., (1996). Properties of a fuzzy relational model. Oxford University Engineering Laboratory Internal Report, OUEL $2119 / 96$.

[12] Wang, L., E. Kannatey-Asibu Jr, et al. (2003). "A method for sensor selection in reconfigurable process monitoring." Journal of Manufacturing Science and Engineering, Transactions of the ASME 125(1): 95-99.

[13] Yager, R.R., Filev, D.P., (1994). Essentials of Fuzzy Modelling and Control, John Wiley, NY.

[14] Zhou Y., Dexter, A. L., Zolotas, A., (2009). "Generating Training Data For Identifying Neuro-fuzzy Models Of Non-Linear Dynamic Systems." The $48^{\text {th }}$ IEEE Conference on Decision and Control and $28^{\text {th }}$ Chinese Control Conference, Shanghai, China

[15] Zhou, Y. (2008). Fault identification in non-linear dynamic systems. University of Oxford. DPhil Thesis 